\title{
High Return to Play Rate and Reduced Career Longevity Following Surgical Management of Athletic Pubalgia in National Basketball Association Players
}

\author{
Joshua P. Castle, M.D., Adam Kessler, B.S., Muhammad J. Abbas, B.S., Susan Wager, B.S., \\ Lafi S. Khalil, M.D., Kelechi R. Okoroha, M.D., and Nima Mehran, M.D.
}

Purpose: To assess the effects of surgical treatment of athletic pubalgia (AP) on game use and performance metrics in National Basketball Association (NBA) players. Methods: A retrospective review of all NBA players who underwent surgical management for AP from 1996 to 2018 was performed. A matched control group was created for comparison. The index period was defined as the entire NBA season in which surgery occurred, including the corresponding offseason. Player demographics, use (games played, games started, and minutes per game) and performance (player efficiency rating) metrics were collected for all players. Statistical analysis was performed to compare data before and after return to play. Results: Thirty players with a history of surgical management for AP were included in the final analysis. Following surgery for AP, NBA players were found to have a return to play (RTP) rate of $90.91 \%$ (30/33). The average RTP following surgery was $4.73 \pm 2.62$ months. Compared with control athletes, athletes in the AP group played significantly fewer seasons postinjury $(4.17 \pm 2.70$ vs $5.49 \pm 3.04$ seasons, respectively; $P=.02)$. During the first year following RTP, NBA players experienced significant reductions in game use and performance, both when compared with the year prior and matched control athletes $(P<.05)$. At 3-year follow-up, players continued to demonstrate significant reductions in game use (minutes per game, $P<.05$ ) but not performance. Conclusions: Following surgical treatment of AP, NBA players demonstrated a high RTP rate, but shortened career. A short-term reduction in game use and performance metrics was found the year of return following surgery. However, 3-year follow-up performance metrics normalized when compared with healthy controls. Study Design: Level III; retrospective case-control study.

$\mathbf{L}$ ower-extremity injuries have been shown to comprise more than $60 \%$ of all injuries in National Basketball Association (NBA) players. ${ }^{1-3}$ Athletic pubalgia (AP), or sports hernia, has become increasingly recognized in athletes over time, often presenting as chronic groin or lower abdominal pain without evidence of a true hernia. ${ }^{4-6}$ Basketball players are particularly at high risk of AP due to the physical demand of the sport requiring rapid cutting, sprinting,

From the Department of Orthopaedic Surgery, Henry Ford Hospital, Detroit, Michigan (J.P.C., M.J.A., L.S.K.); University of California Los Angeles, Los Angeles, California (A.K.); Wayne State University School of Medicine, Detroit, Michigan (S.W.); Department of Orthopedic Surgery, Mayo Clinic, Minneapolis, Minnesota (K.R.O.); and Department of Orthopaedic Surgery, Kaiser Permanente, Los Angeles, California (N.M.), U.S.A.

The authors report the following potential conflicts of interest or sources of funding: N.M. reports other from Arthrex and Smith $\theta$ Nephew, outside the submitted work. K.R.O. reports other from Stryker, Arthrex, Smith $\theta$ Nephew, and Zimmer, outside the submitted work. Full ICMJE author disclosure forms are available for this article online, as supplementary material. sudden acceleration, and deceleration..$^{6-8}$ These physical activities may create imbalanced strain and tension on the muscles and tendons surrounding the pubis and may lead to injury. ${ }^{9,10}$ In a retrospective review of 8490 patients over 2 decades, basketball players were among the top 5 sports affected by AP, comprising $6.2 \%$ of patients. ${ }^{6}$

AP encompasses a broad spectrum of pathologies and includes injury to the posterior inguinal wall, rectus

Received January 13, 2021; accepted July 5, 2021

Address correspondence to Joshua P. Castle, M.D., Department of Orthopaedic Surgery, 2799 W. Grand Blvd., Detroit, MI 48202.E-mail: jcastle1@ hfhs.org

(C) 2021 THE AUTHORS. Published by Elsevier Inc. on behalf of the Arthroscopy Association of North America. This is an open access article under the CC BY-NC-ND license (http://creativecommons.org/licenses/by-nc-nd/4.0/). 2666-061X/2166

https://doi.org/10.1016/j.asmr.2021.07.001 
abdominis insertion, adductor tendons, inguinal nerves, or pubic symphysis. ${ }^{8,11}$ Initial treatment often consists of rest, anti-inflammatories, and physical rehabilitation. ${ }^{9,12}$ For those who do not respond to conservative therapy, surgery is indicated. The wide variety of pathology has led to a diversity of surgical options, ${ }^{6}$ many of which have been shown to have superior outcomes over conservative management. ${ }^{9,13,14}$ Compared with recreational athletes, however, professional athletes endure greater physical demands and may elect to have surgery to return-to-play (RTP) sooner at an elite level. In NBA players, the impact of surgical intervention following RTP is yet to be determined.

While some professional athletes have been shown to successfully RTP at a high level of performance after AP surgical repair, there is limited information available regarding game use and performance in NBA players following RTP. ${ }^{15-17}$ The purpose of this study is to assess the effects of surgical treatment of AP on game use and performance metrics in NBA players. The authors hypothesized that NBA athletes requiring surgical intervention would demonstrate shortened careers but, in the long term, players would maintain game use and performance metrics.

\section{Methods}

A retrospective review of all NBA player who underwent surgical management for AP from 1996 to 2018 was performed. Publicly available injury reports (from espn.com, nba.com, nbcsports.com, etc.), news articles, player profiles, and game previews of starting lineups were used to identify all NBA players who underwent surgical management of AP, similar to previous literature. ${ }^{18-21}$ To verify these reports, at least 2 separate online articles corroborating the injury was deemed sufficient. In addition, the date of surgery was identified, and game statistics were analyzed to confirm missing data following the surgery to confirm consistency with the news reports. For offseason surgeries, 2 articles detailing a reported surgery sufficed for inclusion. Players were excluded if the injury or surgery occurred outside of NBA participation, concomitant injuries were documented, or the player missed significant playing time for other injuries within 2 years before or after the index season, or if the player failed to RTP sufficiently following injury. Failure of RTP was defined as fewer than 15 games in the first season following RTP without substantial increase in seasons thereafter.

The index period was defined as the entire NBA season in which surgery occurred, including the corresponding preseason and offseason, such that the first season following RTP from surgery was considered season +1 . In-game statistical metrics were collected for the 3 seasons prior to the index period and the 3 seasons after RTP. The seasons prior to index were labeled
$-1,-2$, and -3 ; the seasons following RTP were labeled $+1,+2$, and +3 . Player demographics (age, position, height, weight, and body mass index [BMI]), use (games played [GPS], games started [GS], and minutes per game [MPG]) and performance (Player Efficiency Rating [PER]) metrics were collected for all players. Months until return to play (RTP) was determined based on the amount of time passed between date of surgery and the first NBA regular season game indicating RTP. Only regular season NBA games were included in the statistics.

A control group was created for comparison, consisting of a 2:1 control to study group ratio, where controls group players were matched by age, position, BMI, years of experience, era of play, and index season PER. The control index season was chosen so that the age and seasons of experience of the control were similar to the matched player undergoing AP surgical repair. Matching for BMI and PER was allowed within a 5-point range of the respective study subject's BMI and PER. To mitigate differences in the era of play, the index season for the healthy controls was matched within 5 years of the study group players' index season. Years of experience before the index season was within \pm 2 years. $^{18}$

Comparisons between the healthy control group and the surgically treated study group were then conducted for game use and performance statistics before and after the index season in several varieties. Changes in game use and performance were compared between the season directly before the index season and the season directly after RTP for an analysis of direct impact of injury in the short term. In addition, a 3-year comparison was made between the average game use and performance parameters over the 3-year span before the index season and the 3-year span following RTP, to provide an appropriate longer-term representation of these parameters without the acute impact of injury and rehabilitation, similar to previous studies. ${ }^{18,19,22}$ Furthermore, relative percentages of these parameters were calculated for postindex statistics, by creating a "baseline preindex" value derived from a weightedaverage of seasons -1 and -2 . Relative percentages of postindex values were calculated to have a more robust sample of statistics, using methods similar to previous literature. ${ }^{18,23}$ Relative percentages for performance (PER) and game use metrics were calculated for the first season and third season following RTP, and compared between control and study groups, to evaluate for short- and long-term changes in use and performance in relation to each individual players' preinjury baseline. ${ }^{18,23,24}$

\section{Statistical Analysis}

Categorical variables were reported as counts and column percentages, $\mathrm{N}(\%)$, whereas continuous 
Table 3. Game Use and Performance Three Years Before and After Return to Sport

\begin{tabular}{lcc}
\hline Time Point & Hernia $(\mathrm{N}=30)$ & Control $(\mathrm{N}=60)$ \\
\hline GPS & & \\
Pre & $71.20 \pm 13.29$ & $62.53 \pm 19.98$ \\
Post & $59.29 \pm 18.73$ & $63.45 \pm 20.03$ \\
$P$ value: time & .03 & .81 \\
GS & & \\
Pre & $48.68 \pm 27.17$ & $41.14 \pm 29.60$ \\
Post & $35.23 \pm 29.12$ & $39.55 \pm 32.37$ \\
$P$ value: time & .14 & .79 \\
MPG & & \\
Pre & $29.39 \pm 6.98$ & $26.94 \pm 9.68$ \\
Post & $22.99 \pm 8.58$ & $25.71 \pm 8.39$ \\
$P$ value: time & .02 & .49 \\
PER & & $15.77 \pm 4.28$ \\
Pre & $15.77 \pm 4.85$ & $14.56 \pm 5.22$ \\
Post & $12.84 \pm 5.73$ & .21 \\
$P$ value: time & .09 & \\
\hline
\end{tabular}

NOTE. Continuous variables are presented using adjusted mean \pm standard error. $P$ values with significance, $P<.05$, are indicated by bold text.

Time: $P$ value compares pre vs post values within the same group. GPS, games per season; GS, games started; MPG, minutes per game; PER, player efficiency rating.

game use and performance in the year of return compared with matched controls. At 3-year follow-up, game use and performance metrics normalized with the exception of MPG. In addition, players returning from surgical treatment of AP were found to have shorter careers compared with matched controls. These results suggest that NBA players RTP at a high rate after surgical treatment of AP. Game use and performance are most affected in the acute period and the injury may affect overall career length.

Previous literature has highlighted remarkably successful rates of RTP following AP ranging from $80 \%$ to $100 \%$ across multiple sports. ${ }^{6,13,15,16,25-30}$ From 1996 to 2015, 56 National Football League (NFL) players who underwent AP surgery had a RTP rate of $94.7 \% .{ }^{25}$ A cohort of predominately amateur and professional soccer players likewise experienced a $94.4 \%$ RTP rate after laparoscopic inguinal disruption repair. ${ }^{27}$ In the National Hockey League, $80 \%$ of hockey players are reported to successfully RTP after AP repair. ${ }^{26}$ Similar to investigations in other professional arenas, our study demonstrates a high RTP rate of $90.91 \%$ in NBA athletes following surgical treatment of AP. This is in line with previously reported rates exceeding $90 \%$ in other professional sports. Furthermore, a variety of factors beyond the index injury may affect RTP success, such as history of previous injuries, stage in career, trades, and team dynamics.

Successful RTP is not only determined by the ability to play but by the ability to do so at a similar level to preinjury baseline and comparable with other players.
There is conflicting evidence in the literature regarding game use and performance following surgical treatment of AP in professional sports. ${ }^{15,17,25}$ Jack et al. ${ }^{25}$ compared 56 NFL players who underwent surgical treatment of AP to a control group, finding that those in the surgery group played in significantly fewer seasons $(3.8 \pm 2.4$ vs $3.2 \pm 2.1$ years $)$ and GPS ( $14.0 \pm 2.3$ vs $12.0 \pm 3.4)$ after RTP; however, they found no difference in performance between groups. ${ }^{25}$ A retrospective review of NFL combine participants (mean age 22.1 years old) with a history of surgical treatment for AP was conducted by Knapik et al., ${ }^{17}$ who found no significant impact on GPS, GS, draft status, roster activity, or number of seasons played. Our study evaluated NBA players of slightly older age (mean 28.6 years old) and found significantly fewer seasons played following surgical treatment of AP, as well as reduced game use and performance acutely after surgery compared with controls. Interestingly, however, we found that NBA players returned to their baseline use and performance metrics at 3-year follow-up, aside from persistently decreased MPG. Whether their reduced career length or MPG following surgical treatment is attributed to their older age at time of surgery as compared with the NFL cohort requires further study. However, the present findings highlight and support that NBA players have potential to return to full game use and performance capacity in the long-term following surgical treatment of AP.

Following surgical treatment of AP, understanding the appropriate time to RTP is crucial in counseling elite athletes, setting expectations of recovery, and safely returning to full activity. Previous reports of time to RTP following surgical treatment of AP show considerable variation among multiple sports, ranging from as low as 4.23 weeks to beyond 23 weeks. ${ }^{16,29-33}$ This significant variability may be attributed to the inherent ambiguity of the term "sports hernia" or "athletic pubalgia," ${ }^{34}$ which encompasses multiple sites of pathology such as the rectus abdominis, inguinal wall, adductor longus tendon, and pubis symphysis. ${ }^{5,10,35}$ In a systematic review and meta-analysis of inguinal groin pain in athletes of various skill levels from 1980 to 2013 stratified by anatomic sites, King et al. ${ }^{36}$ reported the lowest RTP rates for adductor tendon related pain $(81 \%)$ and highest RTP rates for abdominal pain (96\%). Time to RTP after surgery also varied by location, with pubic and abdominal + adductor pain requiring the longest RTP times (23.1 and 21.9 weeks, respectively) whereas abdominal alone had the shortest ( 7.9 weeks). Kajetanek et al. ${ }^{16}$ similarly demonstrated variable RTP time based on site of pathology, with faster RTP in those having surgery addressing only the abdominal wall as compared with the addition of adductor tenotomies $(91.1 \pm 21$ vs $132.5 \pm 39$ days, $P=.02)$. In the present investigation, we found that NBA players returned to 
A

Fig 1. Relative percentage of use and performance (A) 1 season and (B) 3 seasons postindex. Preindex baseline is represented by the solid line $(100 \%)$ across all variables. Relative percentages below this line indicate that the postindex variable was less than baseline. ${ }^{*} P<.05$ indicates significant differences between athletic pubalgia (AP) surgery and control groups. (GPS, games per season; GS, games started; MPG, minutes per game; PER, player efficiency rating.)

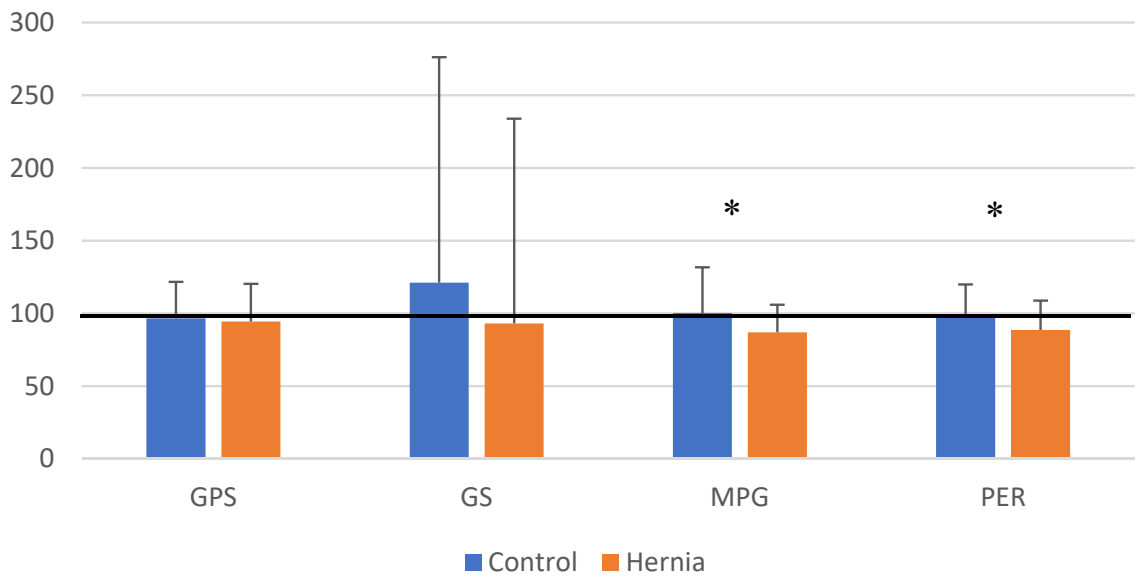

B

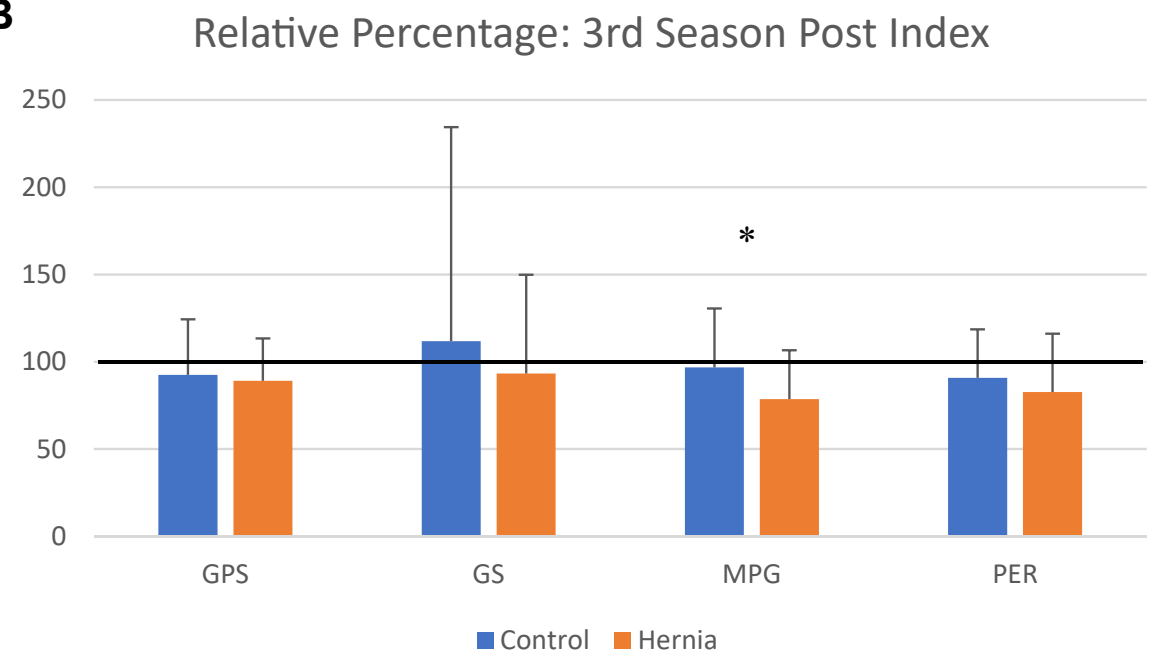

full competition, on average, in $4.73 \pm 2.62$ months, consistent with reports of other elite level athletes. ${ }^{15-17}$ It is important to highlight that AP is a condition treated with a large variety of surgical techniques that may ultimately play a role in varying rates of RTP, time to RTP, and successful return to baseline use and performance metrics.

\section{Limitations}

Our study presents with a variety of limitations. Due to the retrospective design relying on publicly sourced data, details concerning advanced imaging, injury grading, and surgical procedure specifics were unavailable to analyze. Likewise, exact time to RTP based on timing of season and medical clearance is unable to be confirmed. Furthermore, due to the retrospective nature of the study, a power analysis was not performed, and only players with reported cases of the surgical management of AP were included. This could have led to the sample size of players influencing the statistical significance of the analysis performed. As discussed previously, AP includes a spectrum of pathologies and may require multiple types of surgeries. The lack of availability of medical records inherent to this study design prevents our ability to stratify players based on specific pathology, anatomic site, or surgical procedures. In addition, treatment and rehabilitation protocols are likely to have changed throughout the study period. Sampling bias also may occur with publicly reported data due to over-representation and reporting of injuries in more well-known or high performing players. Furthermore, despite excluding players with concomitant injuries, we are unable to definitively exclude commonly associated pathologies such as hip labral tearing and/or femoroacetabular impingement. ${ }^{17,29}$ Future studies should correlate physical examination, 
magnetic resonance imaging, and surgical findings with outcomes after AP repair in terms of workload and performance in these elite basketball players. Outside factors such as coaching, roster composition, and quality of opponents may have played a role in any changes in use and performance, as well. The process of matching controls helps to negate these differences as both the subject and control groups faced this inherent issue of playing in a professional sport similarly.

\section{Conclusions}

Following surgical treatment of AP, NBA players demonstrated a high RTP rate but shortened careers. A short-term reduction in game use and performance metrics was found the year of return following surgery. However, 3-year follow-up performance metrics normalized when compared with healthy controls.

\section{References}

1. Drakos MC, Domb B, Starkey C, Callahan L, Allen AA. Injury in the national Basketball Association: A 17-year overview. Sports Health 2010;2:284-290.

2. Newman JS, Newberg AH. Basketball injuries. Radiol Clin North Am 2010;48:1095-1111.

3. Laver L, Okohara K, Nwachukwu BU, Mei-Dan O, Nho SJ. Hip and groin injuries in basketball. Berlin, Heidelberg: Springer, 2020.

4. Larson CM. Sports hernia/athletic pubalgia: Evaluation and management. Sports Health 2014;6:139-144.

5. Drager J, Rasio J, Newhouse A. Athletic pubalgia (sports hernia): Presentation and treatment. Arthroscopy 2020;36: 2952-2953.

6. Meyers WC, McKechnie A, Philippon MJ, Horner MA, Zoga AC, Devon ON. Experience with "sports hernia" spanning two decades. Ann Surg 2008;248:656-665.

7. Starkey C. Injuries and illnesses in the National Basketball Association: A 10-year perspective. J Athl Train 2000;35: $161-167$.

8. Elattar O, Choi HR, Dills VD, Busconi B. Groin injuries (athletic pubalgia) and return to play. Sports Health 2016;8:313-323.

9. Zuckerbraun BS, Cyr AR, Mauro CS. Groin pain syndrome known as sports hernia: A review. JAMA Surg 2020;155:340-348.

10. Morales-Conde S, Socas M, Barranco A. Sportsmen hernia: What do we know? Hernia 2010;14:5-15.

11. Miller M, Thompson S. DeLee, Drez and Miller's orthopaedic sports medicine. 5th ed. Philadelphia: Elsevier, 2019.

12. Litwin DE, Sneider EB, McEnaney PM, Busconi BD. Athletic pubalgia (sports hernia). Clin Sports Med 2011;30: 417-434.

13. Paajanen H, Brinck T, Hermunen H, Airo I. Laparoscopic surgery for chronic groin pain in athletes is more effective than nonoperative treatment: A randomized clinical trial with magnetic resonance imaging of 60 patients with sportsman's hernia (athletic pubalgia). Surgery 2011;150: 99-107.
14. Muschaweck U, Berger L. Minimal Repair technique of sportsmen's groin: An innovative open-suture repair to treat chronic inguinal pain. Hernia 2010;14:27-33.

15. Gill TJ, Wall AJ, Gwathmey FW, et al. Surgical release of the adductor longus with or without sports hernia repair is a useful treatment for recalcitrant groin strains in the elite athlete. Orthop J Sports Med 2020;8: 2325967119896104.

16. Kajetanek C, Benoit O, Granger B, et al. Athletic pubalgia: Return to play after targeted surgery. Orthop Traumatol Surg Res 2018;104:469-472.

17. Knapik DM, Gebhart JJ, Nho SJ, Tanenbaum JE, Voos JE, Salata MJ. Prevalence of surgical repair for athletic pubalgia and impact on performance in football athletes participating in the National Football League combine. Arthroscopy 2017;33:1044-1049.

18. Khalil LS, Jildeh TR, Tramer JS, et al. Effect of Achilles tendon rupture on player performance and longevity in National Basketball Association players. Orthop J Sports Med 2020;8:2325967120966041.

19. Khalil LS, Matar RN, Rahman T, et al. Effect of workload After ACL reconstruction on rerupture rates in NBA players. Orthop J Sports Med 2020;8:2325967120964467.

20. Tramer JS, Khalil LS, Ziedas A, Mehran N, Okoroha KR. Return to play and performance in the Women's National Basketball Association after anterior cruciate ligament reconstruction. Orthop J Sports Med 2020;8: 2325967120947078.

21. Keller RA, Mehran N, Marshall NE, et al. Major League pitching workload after primary ulnar collateral ligament reconstruction and risk for revision surgery. J Shoulder Elbow Surg 2017;26:288-294.

22. Keller RA, Mehran N, Khalil LS, Ahmad CS, ElAttrache N. Relative individual workload changes may be a risk factor for rerupture of ulnar collateral ligament reconstruction. J Shoulder Elbow Surg 2017;26:369-375.

23. Amin NH, Old AB, Tabb LP, Garg R, Toossi N, Cerynik DL. Performance outcomes after repair of complete Achilles tendon ruptures in National Basketball Association players. Am J Sports Med 2013;41:1864-1868.

24. Trofa DP, Miller JC, Jang ES, Woode DR, Greisberg JK, Vosseller JT. Professional athletes' return to play and performance after operative repair of an Achilles tendon rupture. Am J Sports Med 2017;45:2864-2871.

25. Jack RA, Evans DC, Echo A, et al. Performance and return to sport after sports hernia surgery in NFL players. Orthop J Sports Med 2017;5:2325967117699590.

26. Jakoi A, O'Neill C, Damsgaard C, Fehring K, Tom J. Sports hernia in National Hockey League players: Does surgery affect performance? Am J Sports Med 2013;41:107-110.

27. Piozzi GN, Cirelli R, Salati I, et al. Laparoscopic approach to inguinal disruption in athletes: A retrospective 13-year analysis of 198 patients in a single-surgeon setting. Sports Med Open 2019;5:25.

28. Srinivasan A, Schuricht A. Long-term follow-up of laparoscopic preperitoneal hernia repair in professional athletes. J Laparoendosc Adv Surg Tech A 2002;12:101-106.

29. Gerhardt M, Christiansen J, Sherman B, Miranda A, Hutchinson W, Chahla J. Outcomes following surgical management of inguinal-related groin pain in athletes: A case series. J Hip Preserv Surg 2020;7:103-108. 
30. Emblom BA, Mathis T, Aune K. Athletic pubalgia secondary to rectus abdominis-adductor longus aponeurotic plate injury: Diagnosis, management, and operative treatment of 100 competitive athletes. Orthop J Sports Med 2018;6:2325967118798333.

31. Dojcinovic B, Sebecic B, Staresinic M, Jankovic S, Japjec M, Culjak V. Surgical treatment of chronic groin pain in athletes. Int Orthop 2012;36:2361-2365.

32. Koutserimpas C, Vlasis K, Makris MC, et al. Operative treatment of athletic pubalgia in competitive athletes: A retrospective study. J Sports Med Phys Fitness 2020;60:758-763.

33. de Queiroz RD, de Carvalho RT, de Queiroz Szeles PR, Janovsky C, Cohen M. Return to sport after surgical treatment for pubalgia among professional soccer players. Rev Bras Ortop 2014:49:233-239.

34. Serner A, van Eijck CH, Beumer BR, Holmich P, Weir A, de Vos RJ. Study quality on groin injury management remains low: A systematic review on treatment of groin pain in athletes. Br J Sports Med 2015;49:813.

35. Ekberg O, Persson NH, Abrahamsson PA, Westlin NE, Lilja B. Longstanding groin pain in athletes. A multidisciplinary approach. Sports Med 1988;6:56-61.

36. King E, Ward J, Small L, Falvey E, Franklyn-Miller A. Athletic groin pain: A systematic review and metaanalysis of surgical versus physical therapy rehabilitation outcomes. Br J Sports Med 2015;49:1447-1451. 